\section{Use of Catridecacog in a patient with severe Factor XIII deficiency undergoing surgery}

\author{
Gianluca Sottilotta, ${ }^{1}$ Francesca Luise, ${ }^{1}$ \\ Vincenzo Oriana, ${ }^{1}$ Angela Piromalli, ${ }^{1}$ \\ Rosa Santacroce, ${ }^{2}$ Alessandra Di Lelio ${ }^{3}$ \\ ${ }^{1}$ UOSD Microcitemie - Centro Emofilia \\ - Servizio Emostasi e Trombosi, Grande \\ Ospedale Metropolitano Bianchi- \\ Melacrino-Morelli, Reggio Calabria; \\ ${ }^{2}$ Servizio di Genetica Medica, Azienda \\ Ospedaliero-Universitaria Ospedali \\ Riuniti di Foggia; ${ }^{3}$ Center for Outcomes \\ Research and Clinical Epidemiology - \\ CORESEARCH, Pescara, Italy
}

\begin{abstract}
Despite many articles regarding the antihemorrhagic treatment and prophylaxis, there is a lack of experience about how to best conduct major surgical procedures in patients with congenital factor XIII (FXIII) deficiency.

Here we report a case of surgery (right inguinal hernia, complicated by heaviness and pain) performed in a patient with FXIII deficiency, receiving recombinant FXIII prophylaxis (Catridecacog $35 \mathrm{UI} / \mathrm{kg}$ every $28 \pm 2$ days). Our experience shows that Catridecacog can be used safely and effectively not only for continued prophylaxis but also in surgery and adds to the very limited body of evidence currently available on surgery in this bleeding disorder.
\end{abstract}

\section{Introduction}

Factor XIII (FXIII) is the terminal enzyme in the blood coagulation cascade and essential for cross-linking fibrin molecules to form an effective and stable clot. In plasma, FXIII circulates as an inactive heterotetramer composed of two catalytic FXIII A-subunits and two carrier FXIII Bsubunits (FXIII- $\left.\mathrm{A}_{2} \mathrm{~B}_{2}\right)^{1,}{ }^{1,2} \mathrm{~A}$ deficiency or dysfunction of either the A or B subunit can result in FXIII deficiency. Congenital FXIII deficiency is a rare and serious autosomal recessive coagulation disorder with a high risk of life-threatening bleeding complications. ${ }^{3}$ The prevalence is estimated to be approximately 1 in 1 to 2 million people worldwide. ${ }^{4,5}$ Patients with congenital FXIII deficiency experience major spontaneous bleeding episodes more frequently than patients with most other rare bleeding disorders ${ }^{4}$ and are at high risk of intraoperative and delayed postoperative bleeds. ${ }^{3}$ These patients require FXIII replacement to treat bleeds and, even more importantly, to prevent them (prophylaxis).

Until recently, only plasma-derived sources of FXIII have been available (fresh frozen plasma, cryoprecipitate and plasmaderived FXIII concentrate). Nowadays, a recombinant factor XIII (rFXIII) is available: Catridecacog is efficient in patients with congenital factor XIII A-subunit deficiency: a dose of $35 \mathrm{UI} / \mathrm{kg}$ every $28 \pm 2$ days is recommended for prophylaxis ${ }^{6}$ and a trough level of greater than 5\% FXIII activity should be aimed for. ${ }^{7}$

\section{Case Report}

We report a case of a Caucasian 47year-old male patient with severe FXIII subunit A congenital deficiency (FXIII $<1 \%$ ). The genetic mutation which caused FXIII deficit is Gly563Arg in homozygosity. He has two sisters with the same bleeding disorder and the same level of severity.

His disease was diagnosed at the age of 4 years after a surgery for left inguinal hernia complicated by postoperative hemorrhage with need for blood transfusion.

This patient had been in prophylaxis first with FXIII plasma concentrate (1250 UI every 21 days from November 1985 to October 1987). It was then withdrawn from the market, so he was treated with fresh frozen plasma in prophylaxis every 21 days (from November 1987 to October 2013).

Then, the patient restarted prophylaxis with FXIII plasma concentrate 1500 UI (18 $\mathrm{UI} / \mathrm{kg}$ ) since this drug was reintroduced. From July $22^{\text {nd }}, 2014$, when the patient was 44 years old, he started prophylaxis with rFXIII: Catridecacog $30 \mathrm{UI} / \mathrm{kg}$ every 30 days. That was the first time that Catridecacog was administered in Italy.

During the prophylaxis period with Catridecacog, the patient did not report any hemorrhagic episode even in work-related traumas (except for a post-traumatic subcutaneous hematoma, which regressed after three days from the treatment administration). Prophylaxis was successful, without adverse events. A right inguinal hernia, complicated by heaviness and pain, was diagnosed in July 2017. Because of the risk of a strangulated hernia or obstruction, the patient was scheduled to have surgery. The surgery was performed 2 weeks after the last prophylaxis infusion: the pre-operative FXIII dosage showed levels of $21.5 \%$ (immunoassay). Catridecacog $29.7 \mathrm{UI} / \mathrm{Kg}$ was infused 4 hours before surgery.
Correspondence: Alessandra Di Lelio, Center for Outcomes Research and Clinical Epidemiology - CORESEARCH, Via Tiziano Vecellio 2, 65124 Pescara, Italy.

Tel.: +39.085.9047114 - Fax: +39.085.9047113. E-mail: dilelio@coresearch.it

Key words: Recombinant FXIII, congenital FXIII deficiency, surgery.

Acknowledgements: The editorial assistance was provided by Airon Communication through a Novo Nordisk S.p.A. unconditional grant. The authors of the publication are fully responsible for the contents and conclusions. Novo Nordisk S.p.A. did not influence and has not been involved in the data interpretation and statistical analysis presented in the manuscript.

Contributions: GS, FL, VO, AP, RS: substantial contributions to conception and design, drafting and writing the case report; final approval of the version to be published. ADL: drafting and writing the case report, final approval of the version to be published. GS is the guarantor of this work, accountable for all aspects of the work in ensuring that questions related to the accuracy or integrity of any part of the work are appropriately investigated and resolved.

Conflict of interest: the authors declare no potential conflict of interest.

Funding: None

Received for publication: 17 October 2018. Accepted for publication: 17 January 2019.

This work is licensed under a Creative Commons Attribution-NonCommercial 4.0 International License (CC BY-NC 4.0).

(C) Copyright G. Sottilotta, et al., 2019

Licensee PAGEPress, Italy

Hematology Reports 2019; 11:7912

doi:10.4081/hr.2019.7912

Dosages for FXIII showed a $50.7 \%$ and $41.1 \%$ level at 39 and 66 hours after surgery, respectively (immunoassay). No intraoperative or post-surgical bleeding occurred. Hemoglobin levels were available before $(13.6 \mathrm{~g} / \mathrm{dL})$ and after surgery (12.2 $\mathrm{g} / \mathrm{dL})$. No other intraoperative or postoperative complications occurred. No further administration of Catridecacog or any other anti-hemorrhagic drug was required.

The patient was discharged 3 days after the surgery. In the postoperative period, no hemorrhage or surgical wound healing delay occurred. After the surgery, the patient regularly resumed his prophylactic program every 30 days. 


\section{Discussion}

Little is known about how to best conduct major surgical procedures in patients with congenital FXIII deficiency.

To date, the only published data on the effectiveness of rFXIII in the surgical setting derive from the Mentor ${ }^{\mathrm{TM}} 2$ trial, a trial on efficacy and safety of monthly replacement therapy with rFXIII. As elective surgery was permitted, the trial provided the first data on minor surgical procedures performed in patients receiving rFXIII prophylaxis. All 12 minor procedures were performed within 1 to 21 days of the last scheduled rFXIII dose ( 8 procedures were performed within 7 days of patient's last scheduled dose and 4 were performed 10 to 21 days after the dose) and additional FXIII substitution was not administered for any of these procedures These findings suggest that prophylaxis with $35 \mathrm{UI} / \mathrm{kg}$ rFXIII every 4 weeks provides sufficient hemostatic coverage for the perioperative management of minor surgical procedures in patients with congenital FXIII deficiency, without the need for additional FXIII treatment. ${ }^{6}$

In our case, despite the pre-surgery FXIII level was above the hemostatic threshold, we decided to infuse Catridecacog to prevent bleedings because of the well-known FXIII half-life reduction in consumptive processes such as surgery. Only one infusion was sufficient to achieve the anti-hemorrhage efficacy as was also evidenced by elevated FXIII levels even three days after the surgery. No other antihemorrhagic therapy was required.

\section{Conclusions}

Our case report shows that Catridecacog can be used safely and effectively not only for continued prophylaxis ${ }^{7}$ but also in surgery and add to the very limited body of evidence currently available on surgery in FXIII-deficiency patients.

\section{References}

1. Chung SI, Lewis MS, Folk JE. Relationships of the catalytic properties of human plasma and platelet transglutaminases (activated blood coagulation factor XIII) to their subunit structures. J Biol Chem 1974;249:94050.

2. Schwartz ML, Pizzo SV, Hill RL, McKee PA. Human factor XIII from plasma and platelets. Molecular weights, subunit structures, proteolytic activation, and cross-linking of fibrinogen and fibrin. J Biol Chem 1973;248:1395-407.

3. Muszbek L, Katona É. Diagnosis and Management of Congenital and Acquired FXIII Deficiencies. Semin Thromb Hemost 2016;42:429-39.

4. Peyvandi F, Palla R, Menegatti M, et al. European Network of Rare Bleeding Disorders Group. Coagulation factor activity and clinical bleeding severity in rare bleeding disorders: results from the European Network of Rare Bleeding Disorders. J Thromb Haemost 2012;10: 615-21.

5. Anwar R, Minford A, Gallivan L, et al. Delayed umbilical bleeding: a presenting feature for factor XIII deficiency: clinical features, genetics, and management. Pediatrics 2002; 109:E32.

6. Carcao M, Altisent C, Castaman G, et al. Recombinant FXIII (rFXIII-A(2)) Prophylaxis Prevents Bleeding and Allows for Surgery in Patients with Congenital FXIII A-Subunit Deficiency. Thromb Haemost 2018;118: 451-60.

7. Muszbek L, Katona É. Diagnosis and Management of Congenital and Acquired FXIII Deficiencies. Semin Thromb Hemost 2016;42:429-39. 